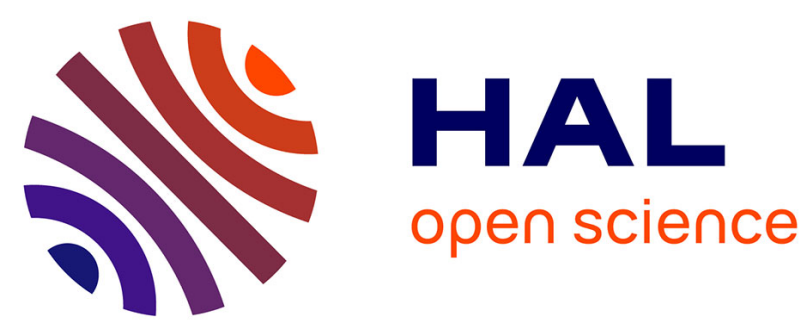

\title{
Role of Correlations in the Collective Behavior of Microswimmer Suspensions
}

Joakim Stenhammar, Cesare Nardini, Rupert W Nash, Davide Marenduzzo, Alexander A Morozov

\section{- To cite this version:}

Joakim Stenhammar, Cesare Nardini, Rupert W Nash, Davide Marenduzzo, Alexander A Morozov. Role of Correlations in the Collective Behavior of Microswimmer Suspensions. Physical Review Letters, 2017, 119, pp.028005. 10.1103/PhysRevLett.119.028005 . cea-01687396

\section{HAL Id: cea-01687396 https://hal-cea.archives-ouvertes.fr/cea-01687396}

Submitted on 18 Jan 2018

HAL is a multi-disciplinary open access archive for the deposit and dissemination of scientific research documents, whether they are published or not. The documents may come from teaching and research institutions in France or abroad, or from public or private research centers.
L'archive ouverte pluridisciplinaire HAL, est destinée au dépôt et à la diffusion de documents scientifiques de niveau recherche, publiés ou non, émanant des établissements d'enseignement et de recherche français ou étrangers, des laboratoires publics ou privés. 


\title{
Role of Correlations in the Collective Behavior of Microswimmer Suspensions
}

\author{
Joakim Stenhammar, ${ }^{1, *}$ Cesare Nardini, ${ }^{2,3,4, \dagger}$ Rupert W. Nash, ${ }^{5}$ Davide Marenduzzo, ${ }^{3}$ and Alexander Morozov ${ }^{3, \$}$ \\ ${ }^{1}$ Division of Physical Chemistry, Lund University, P.O. Box 124, S-221 00 Lund, Sweden \\ ${ }^{2}$ DAMTP, Centre for Mathematical Sciences, Wilberforce Road, Cambridge CB3 OWA, United Kingdom \\ ${ }^{3}$ SUPA, School of Physics and Astronomy, The University of Edinburgh, \\ James Clerk Maxwell Building, Peter Guthrie Tait Road, Edinburgh EH9 3FD, United Kingdom \\ ${ }^{4}$ Service de Physique de l'État Condensé, CNRS UMR 3680, CEA-Saclay, 91191 Gif-sur-Yvette, France \\ ${ }^{5}$ EPCC, James Clerk Maxwell Building, Peter Guthrie Tait Road, Edinburgh EH9 3FD, United Kingdom
}

(Received 1 February 2017; published 13 July 2017)

\begin{abstract}
In this Letter, we study the collective behavior of a large number of self-propelled microswimmers immersed in a fluid. Using unprecedentedly large-scale lattice Boltzmann simulations, we reproduce the transition to bacterial turbulence. We show that, even well below the transition, swimmers move in a correlated fashion that cannot be described by a mean-field approach. We develop a novel kinetic theory that captures these correlations and is nonperturbative in the swimmer density. To provide an experimentally accessible measure of correlations, we calculate the diffusivity of passive tracers and reveal its nontrivial density dependence. The theory is in quantitative agreement with the lattice Boltzmann simulations and captures the asymmetry between pusher and puller swimmers below the transition to turbulence.
\end{abstract}

DOI: 10.1103/PhysRevLett.119.028005

A suspension of particles that can extract energy from their surroundings and transform it into self-propulsion is an archetypal example of active matter [1,2]. Such systems do not obey the principle of detailed balance [3] at a singleparticle level and their behavior often differs significantly from that of passive suspensions at the same conditions [4]. Experiments on self-propelled particles, such as bacteria [5-7], sperm cells [8], mixtures of microtubules and molecular motors [9], vibrated granular rods [10], "Quincke rollers" [11], and "colloidal surfers" [12], reveal the existence of nonequilibrium steady states with nonzero macroscopic fluxes in these systems. One of the most striking examples is the phenomenon of "bacterial turbulence" [7,13-15], whereby a suspension of swimming bacteria at sufficient density forms a state with large-scale, coherent fluid motion.

Previous analytical [16-19] and numerical [20-24] studies recognize long-range hydrodynamic interactions between swimmers as a key ingredient of their collective motion; in the absence of external forces and torques, these interactions can be described by a dipolar field [25]. The main observation of the previous studies is that bacterial turbulence only emerges in suspensions of pushers (dipolar swimmers that expel fluid along their long direction) and is absent for pullers (dipolar swimmers that do the opposite). This conclusion has been corroborated by mean-field kinetic theories that consider the dynamics of a single swimmer in an average hydrodynamic field produced by other particles [16-19,24].

Below the transition to collective motion, previous theoretical studies view motile suspensions as random and featureless, only acquiring nontrivial properties above the transition. In this Letter, we demonstrate that due to the longrange nature of (unscreened) hydrodynamic interactions between swimmers, pretransitional suspensions develop very strong correlations that dominate their dynamical properties (see also [26]). Strong spatial, temporal, and orientational correlations between swimmers act as precursors to bacterial turbulence and are essential to understanding the transition. We therefore develop a novel kinetic theory that goes beyond mean field and compare its predictions to particle-resolved lattice Boltzmann (LB) simulations of up to $4 \times 10^{6}$ hydrodynamically interacting microswimmers.

To illustrate the significance of swimmer-swimmer correlations, we consider the advection of passive tracer particles immersed in a microswimmer suspension. The effective tracer diffusion constant is an observable sensitive to the dynamical state of the system, and it has been extensively studied experimentally in suspensions of bacteria [27-29] and algae [30-32]. For low densities of swimmers, it has been predicted to scale linearly with the swimmer density and to be identical for pushers and pullers of equal dipolar strengths [28,29,33-36]. Here, we use LB simulations and kinetic theory to show how correlations break the pusher-puller symmetry and result in nonlinear scaling of the enhanced diffusivity with swimmer density. Our analysis suggests that these correlations become significant even for densities as low as $10 \%$ of the critical density, with the latter being estimated at a volume fraction of $\sim 2 \%$ for $E$. coli-like parameters (see below).

Model description.-We consider a three-dimensional suspension of $N$ microswimmers immersed in a fluid of volume $V$ at number density $n=N / V$. Each swimmer exerts a force on the fluid, $-F \mathbf{p}$, representing the flagellum, and an equal and opposite force, $F \mathbf{p}$, representing the cell body, applied a distance $l$ from the propulsive force; here $\mathbf{p}$ is the swimmer orientation [see Fig. 1(a)]. The body is modeled as a sphere with hydrodynamic radius $a$, while the velocity scale 
(a)

(b)

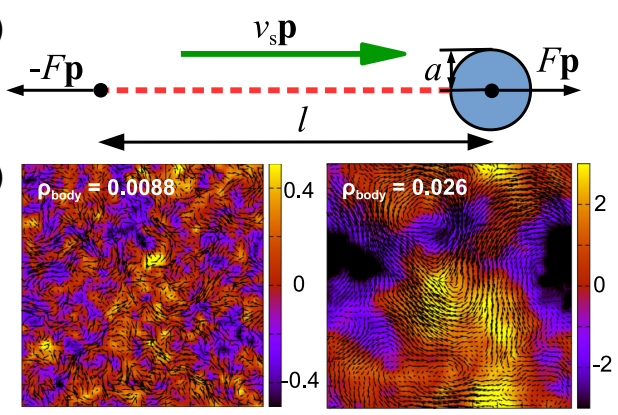

(c)

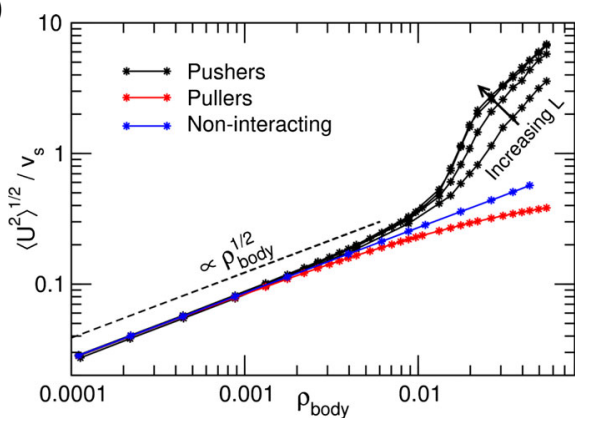

FIG. 1. (a) Schematic picture of the model and its parameters for a pusher swimmer. (b) Snapshots showing the fluid velocity (in units of $v_{s}$ ) from LB simulations of pusher suspensions below (left) and above (right) the transition to turbulence. The vectors denote the in-plane fluid velocity, while the color map shows the out-of-plane component. (c) Root-mean-square fluid velocity $\left\langle U^{2}\right\rangle^{1 / 2}$ from LB simulations. The pusher results are presented for four different side lengths of the cubic LB box: $L=25,50$, 100 , and 200 in LB units (see [37]); other curves are for $L=100$. All densities are given in units of the swimmer body volume fraction $\rho_{\text {body }}=(4 \pi / 3) a^{3} n$, with $a \approx 0.3$ (see [37]).

of this model is defined as $v_{0} \equiv F /(\mu l)$, where $\mu$ is the viscosity of the fluid. The dipolar strength of each swimmer is given as $\kappa=F l / \mu$, with $\kappa>0$ representing pushers and $\kappa<0$ representing pullers, and the nondimensional swimmer density is defined as $\rho=n l^{3}$. To facilitate comparison with experimental results, we also define the reduced density $\rho_{\text {body }}=(4 \pi / 3) a^{3} n$, where $a$ is estimated as described in [37], which gives an estimate of the volume fraction based on the bacterial body volume. Each swimmer $i$ moves according to the following equations of motion:

$$
\dot{r}_{i}^{\alpha}=v_{s} p_{i}^{\alpha}+U^{\alpha}\left(\mathbf{r}_{i}\right), \quad \dot{p}_{i}^{\alpha}=\mathbb{P}_{i}^{\alpha \beta} \nabla_{i}^{\gamma} U^{\beta}\left(\mathbf{r}_{i}\right) p_{i}^{\gamma},
$$

where $\mathbb{P}_{i}^{\alpha \beta}=\delta_{\alpha \beta}-p_{i}^{\alpha} p_{i}^{\beta}, \mathbf{U}\left(\mathbf{r}_{i}\right)$ is the fluid velocity at the position of swimmer $i$, and $v_{s}$ is the swimming speed (see [37]); Greek indices denote Cartesian components. In addition to being rotated by the fluid, the orientation of each swimmer is randomized with average tumbling frequency $\lambda$ [38].

Lattice Boltzmann simulations.-Large-scale numerical simulations of up to $\mathcal{O}\left(10^{6}\right)$ hydrodynamically interacting microswimmers in cubic boxes with periodic boundary conditions were performed using a D3Q15 LB algorithm.

The swimmers are described using the point-force implementation developed by Nash et al. [39,40], which accurately captures the full far-field interactions between the particles while neglecting short-range hydrodynamics, lubrication effects, and nonhydrodynamic interactions; see [37] for further details. As shown in Fig. 1(b), we qualitatively capture the transition from seemingly random motion at low density of swimmers to bacterial turbulence at higher densities of pushers, visible as large-scale fluid vortices and jets. Quantitatively, this is characterized in Fig. 1(c) as a rapid deviation of the root-mean-square fluid velocity $\left\langle U^{2}\right\rangle^{1 / 2}$ for pushers at $\rho_{\text {body }} \approx 0.02$ (i.e., close to the experimentally observed transition densities [7,13]), from the expected $\rho^{1 / 2}$ behavior at low density to a state with much larger velocity fluctuations; for pullers, correlations lead to $\left\langle U^{2}\right\rangle^{1 / 2}$ increasing slower than $\rho^{1 / 2}$. Figure 1(c) also highlights the strong system-size dependence of these results: velocity fluctuations change appreciably when going from a box length of $L=25\left(N \simeq 10^{3}-10^{4}\right.$, comparable to previous particleresolved studies [22-24]) to $L=200\left(N \simeq 10^{6}-10^{7}\right)$, indicating the need for very large-scale simulations (at least $L=100$ and $N \simeq 10^{5}$ ) to describe the collective motion in these systems. We postpone further characterization of the turbulent state to future studies, and in the following we focus on velocity fluctuations in the pretransitional region $\left(\rho_{\text {body }} \leq 0.02\right)$ and the buildup of correlations that lead to bacterial turbulence.

In Fig. 2, we make the observation that, in this density regime, interactions between swimmers are dominated by their mutual rotation while the effect of advection is secondary. There we also show results obtained for socalled shakers - particles that apply forces to the fluid but do not swim. These behave very similarly to swimmers, not only qualitatively [41] but also quantitatively, indicating

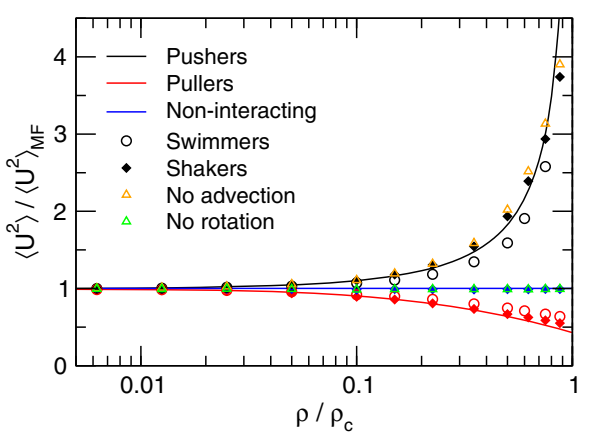

FIG. 2. Variance of the fluid velocity as obtained from LB simulations (symbols) and kinetic theory (lines), normalized by its mean-field value $\left\langle U^{2}\right\rangle_{\mathrm{MF}}=21 v_{0} \rho \kappa_{n}^{2} /\left(2048 \epsilon_{n}\right)$. The density is normalized by the critical density $\rho_{c}=5 \lambda_{n} /\left|\kappa_{n}\right|$. The blue line shows the mean-field prediction, which matches simulations when swimmer-swimmer interactions are switched off. Orange and green triangles are LB results for pusher shakers without either advection or rotation by the fluid. The theoretical results were obtained by numerically solving the full integrals given before Eq. (8). 
that the effect of swimming is subdominant. We now proceed to develop the kinetic theory, where the latter observation will prove to be mathematically convenient.

Kinetic theory.-The central theoretical tool developed in this Letter is a kinetic theory that describes the suspension at a coarse-grained level. We assume that the swimmer density $\rho$ is sufficiently low that the singleswimmer Fourier-space velocity field $\mathbf{u}_{\mathbf{k}}(\mathbf{p})$ can be described by that of a regularized point dipole [42]:

$$
k^{2} u_{\mathbf{k}}^{\alpha}(\mathbf{p})=-i \kappa A(k \epsilon)(\mathbf{k} \cdot \mathbf{p})\left[p_{\alpha}-k^{-2} k_{\alpha}(\mathbf{k} \cdot \mathbf{p})\right] .
$$

Here, $k=|\mathbf{k}|, A(x)=x^{2} K_{2}(x) / 2$ with $K_{2}$ the modified Bessel function of the second kind, and $\epsilon$ is a regularization parameter. The starting point of any kinetic theory is standard [43] and is briefly summarized in the following. The dynamics of the system is described by the master equation [44] for the $N$-body probability density function (PDF) $f_{N}\left(\mathbf{z}_{1}, \ldots, \mathbf{z}_{N}, t\right)$, with $\mathbf{z}_{i}=\left(\mathbf{r}_{i}, \mathbf{p}_{i}\right)$. We then introduce the reduced PDFs $f_{s}=N ! /(N-s) ! \int d \mathbf{z}_{s+1} \cdots d \mathbf{z}_{N} f_{N}$ and derive a BBGKY hierarchy for the $f_{s}$ [37]. This hierarchy is conveniently written in terms of connected correlations $g_{s}$ defined by $f_{2}\left(\mathbf{z}_{1}, \mathbf{z}_{2}, t\right)=f_{1}\left(\mathbf{z}_{1}, t\right) f_{1}\left(\mathbf{z}_{2}, t\right)+g_{2}\left(\mathbf{z}_{1}, \mathbf{z}_{2}, t\right)$ and similarly for higher orders. To close the exact BBGKY hierarchy in a controlled way, we follow the approximation scheme used in equilibrium $[43,45-48]$ and nonequilibrium $[49,50]$ systems with long-range interactions, such as plasmas and self-gravitating systems. We consider the limit of a large number of swimmers, $N \gg 1$, at a fixed density $\rho$; our approach is perturbative in the small parameter $1 / N$, not in $\rho$, and, for this reason, we get accurate predictions even close to the onset of bacterial turbulence. In this limit, and in the absence of hidden divergences [51], we can demonstrate that $f_{1} \sim \mathcal{O}(1)$ and $g_{s} \sim \mathcal{O}\left(1 / N^{s-1}\right)$. At leading order, $g_{s}=0$ for $s \geq 2$, we obtain the mean-field approximation already analyzed in the literature for swimmers [16-19,24] and suspensions of passive rods [52]. In this Letter, we go beyond the mean-field approximation, retaining $g_{2}$ and discarding $g_{s}$ for $s \geq 3$ [37]; henceforth, we use $f \equiv f_{1}$ and $g \equiv g_{2}$.

The perturbative analysis summarized above shows that $g$ solves

$$
\partial_{t} g+L_{f}^{(1)}[g]+L_{f}^{(2)}[g]=C,
$$

where [53]

$$
\begin{aligned}
C \equiv & 3\left(\frac{n}{4 \pi}\right)^{2}\left[p_{1}^{\alpha} p_{1}^{\beta} \nabla^{\alpha} u^{\beta}\left(\mathbf{r}_{1}-\mathbf{r}_{2}, \mathbf{p}_{2}\right)\right. \\
& \left.+p_{2}^{\alpha} p_{2}^{\beta} \nabla^{\alpha} u^{\beta}\left(\mathbf{r}_{2}-\mathbf{r}_{1}, \mathbf{p}_{1}\right)\right],
\end{aligned}
$$

and

$$
\begin{aligned}
L_{f}[h]= & \lambda h-\frac{\lambda}{4 \pi} \int d \mathbf{p}^{\prime} h+\nabla^{\alpha}\left(v_{s} p^{\alpha} h\right) \\
& +\frac{n}{4 \pi} \partial^{\alpha}\left(\mathbb{P}^{\alpha \beta} \nabla^{\gamma} U_{\mathrm{MF}}^{\beta}[h] p^{\gamma}\right),
\end{aligned}
$$

where we used the notation $\partial^{\alpha} \equiv \mathbb{P}^{\alpha \beta}\left(\partial / \partial p^{\beta}\right)$. Here, $U_{\mathrm{MF}}^{\alpha}[h](\mathbf{r})=\int d \mathbf{r}_{1} d \mathbf{p}_{1} u^{\alpha}\left(\mathbf{r}-\mathbf{r}_{1}, \mathbf{p}_{1}\right) h\left(\mathbf{r}_{1}, \mathbf{p}_{1}, t\right)$ and $L_{f}[h]$ is the mean-field operator $V$ linearized close to $f$ and acting on the function $h$, where $V=\nabla^{\alpha}\left(\dot{r}_{\mathrm{MF}}^{\alpha} f\right)+\partial^{\alpha}\left(\dot{p}_{\mathrm{MF}}^{\alpha} f\right)+$ $\lambda f-(\lambda / 4 \pi) \int d \mathbf{p} f$ and $\left(\dot{\mathbf{r}}_{\mathrm{MF}}, \dot{\mathbf{p}}_{\mathrm{MF}}\right)$ are given by Eqs. (1) with $U^{\alpha}\left(\mathbf{r}_{i}\right)$ replaced by $U_{\mathrm{MF}}^{\alpha}[f](\mathbf{r}) ; L_{f}^{(i)}$ acts on $\mathbf{z}_{i}$.

Motivated by our numerical observation that the nature of the transition and the properties of the suspension can be understood in the absence of self-propulsion, we consider only the case of $v_{s}=0$. We stress that, while being significantly more complex, all the results presented below can also be obtained for $v_{s}>0$, as will be shown in a forthcoming publication [54].

We now introduce an Itō white noise $\eta$ with covariance $\mathbb{E}[\eta \eta]=C \delta\left(t-t^{\prime}\right)$, where $\mathbb{E}$ denotes the average over $\eta$, and $C$ is given by Eq. (4). Formally, the connected correlator $g$ can be written as $g\left(\mathbf{z}_{1}, \mathbf{z}_{2}, t\right)=\mathbb{E}\left[\delta f\left(\mathbf{z}_{1}, t\right) \delta f\left(\mathbf{z}_{2}, t\right)\right]$, where $\delta f$ solves

$$
\partial_{t} \delta f+L_{f}[\delta f]=\eta .
$$

Because $g$ is the covariance of density fluctuations close to $f$, these fluctuations are described by the random field $\delta f$. Being small, they are given by a linear stochastic process, Eq. (6), although the variance of the noise $C$ is nontrivial and could not have been guessed a priori. It is also remarkable that, as a result of the coarse graining procedure, the density fluctuations are described by a stochastic process even when the underlying microscopic dynamics are deterministic $(\lambda=0)$. Equations (3) and (6) can be solved exactly to yield $\delta f=e^{-t L_{f}} \delta f(t=0)+$ $\int_{0}^{t} e^{-(t-s) L_{f}} \eta(s) d s$ and $g=\int_{0}^{t} e^{-s L_{f}^{(1)}} e^{-s L_{f}^{(2)}} C d s$ [55]. As $\tilde{\delta f}=e^{-t L_{f}} \tilde{\delta f}(t=0)$ solves $\partial_{t} \tilde{\delta f}+L_{f}[\tilde{\delta f}]=0$, to compute $g$ we only need the solution $\tilde{\delta f}$ of the above deterministic dynamics with appropriate initial conditions set by $C$. It turns out that $\tilde{\delta f}$ can be found exactly for a generic initial condition [37]. For the fluctuations of the fluid velocity in the Fourier-Laplace space $\tilde{\delta} \tilde{U}_{\mathbf{k}}^{\alpha}(\omega)=\int d^{2} \mathbf{p}^{\prime} u_{\mathbf{k}}^{\alpha}\left(\mathbf{p}^{\prime}\right) \tilde{\delta f_{\mathbf{k}}}\left(\mathbf{p}^{\prime}, \omega\right)$, we then obtain the closed expression

$$
\delta \tilde{U}_{\mathbf{k}}^{\alpha}(\omega)=\frac{1}{C_{0}(k, \omega)} \int d^{2} \mathbf{p} \frac{\tilde{\delta f_{\mathbf{k}}}(\mathbf{p}, t=0) u_{\mathbf{k}}^{\alpha}(\mathbf{p})}{-i \omega+\lambda},
$$

where, for the regularized dipolar field in Eq. (2), we have $C_{0}(k, \omega)=1-(\kappa n / 5) A(k \epsilon) /(-i \omega+\lambda)$. Equation (7) is valid under the assumption that the dynamical state described by $f$ is linearly stable, which corresponds to the zeros $\omega_{*}=\omega_{R}+i \omega_{I}$ of $C_{0}(k, \omega)$ having negative imaginary parts in the Laplace domain, $\omega_{I}<0$. These zeros are given by $\omega_{R}=0$ and $\omega_{I}(k \epsilon)=-\lambda+\kappa n A(k \epsilon) / 5$, which implies that a suspension of pullers $(\kappa<0)$ is always stable, while pusher suspensions $(\kappa>0)$ are stable only if $n<5 \lambda / \kappa$, in agreement with earlier results [16-19]. 
We note the emergence of the characteristic time scale $\omega_{I}^{-1}$, which describes the typical time for a small fluctuation of the fluid velocity to relax. At $\rho=0$, it reduces to $\omega_{I}^{-1}=\lambda^{-1}$, while upon increasing the density, it decreases for pullers and increases for pushers. This suggests qualitative differences between the statistical properties of suspensions of pushers and pullers even below the onset of bacterial turbulence.

Fluid velocity variance.-We now compare the kinetic theory developed above with the results of LB simulations by computing the variance $\left\langle U^{2}\right\rangle$ of the fluid velocity: $\left\langle U^{2}\right\rangle=\left\langle U^{2}\right\rangle_{\mathrm{MF}}+\left\langle U^{2}\right\rangle_{\text {corr }}$, where $\left\langle U^{2}\right\rangle_{\mathrm{MF}}=n /\left(4 \pi(2 \pi)^{3}\right) \times$ $\int d \mathbf{k} d \mathbf{p} u_{\mathbf{k}}^{\alpha}(\mathbf{p}) u_{-\mathbf{k}}^{\alpha}(\mathbf{p})$ is the mean-field contribution and $\left\langle U^{2}\right\rangle_{\text {corr }}$ contains corrections induced by swimmer-swimmer correlations. Using the formal solution for $g$ and Eqs. (4) and (7), we obtain $\left\langle U^{2}\right\rangle_{\mathrm{MF}}=v_{0}^{2}\left(\rho \kappa_{n}^{2} / 15 \pi^{2}\right) \int_{0}^{\infty} d k A^{2}\left(\epsilon_{n} k\right)$ and $\left\langle U^{2}\right\rangle_{\text {corr }}=-v_{0}^{2}\left(\rho^{2} \kappa_{n}^{3} / 75 \pi^{2}\right) \int_{0}^{\infty} d k\left[A^{2}\left(\epsilon_{n} k\right) / \omega_{n}\left(\epsilon_{n} k\right)\right]$, which is well approximated by [37]

$$
\frac{\left\langle U^{2}\right\rangle}{v_{0}^{2}} \approx \frac{21 \rho \kappa_{n}^{2}}{2048 \epsilon_{n}}\left(1 \pm \frac{\rho\left(2 \rho_{c} \mp \rho\right)}{2 \rho_{c}\left(\rho_{c} \mp \rho\right)}\right),
$$

where the upper sign corresponds to pushers and the bottom sign to pullers. Here, we have introduced the non-dimensional units $\quad \kappa_{n}=\kappa /\left(l^{2} v_{0}\right), \quad \epsilon_{n}=\epsilon / l, \quad \omega_{n}=\omega_{I} l / v_{0}, \quad$ and $\lambda_{n}=\lambda l / v_{0}$, using $l$ and $l / v_{0}$ as the respective length and time scales. The density $\rho_{c} \equiv 5 \lambda_{n} /\left|\kappa_{n}\right|$ corresponds to the onset of collective motion for pushers, and acts as a characteristic density scale for pullers. Figure 2 shows excellent agreement between this prediction and the LB data, even close to the onset of turbulence, emphasizing the nonperturbative nature of our approach. Equation (8) and the data in Fig. 2 can also be used to assess the relative importance of correlations: for $\rho \lesssim 0.1 \rho_{c}$, pushers and pullers behave equivalently and follow the meanfield prediction, while above it correlations have to be taken into account to obtain correct quantitative predictions. We note furthermore that, using the body volume $(4 \pi / 3) a^{3}$ with $a \approx 0.3$ [37], we obtain $\rho_{c}^{\text {body }} \approx 0.023$, in good agreement with the experimentally observed transitional volume fractions of $2 \%$ in E. coli [7] and B. subtilis [13].

Enhanced diffusivity.-We now consider the advection of a passive tracer with the dynamics $\dot{\mathbf{x}}=\mathbf{U}(\mathbf{x}, t)$ immersed in a suspension of shakers. Its long-time motion is diffusive [56], and we use the kinetic theory developed here to calculate its effective diffusion constant $D_{h}$. Denoting by $\mathbf{x}_{t}$ the tracer position at time $t$, we have $\left\langle\left|\mathbf{x}_{t}-\mathbf{x}_{0}\right|^{2}\right\rangle=6 D_{h} t$, with $D_{h}$ related to the fluid velocity autocorrelation by [57]

$$
D_{h}=\frac{1}{3} \int_{0}^{\infty} d s \mathcal{C}\left(\mathbf{x}_{s}, s, \mathbf{x}_{0}, 0\right) .
$$

Here, $\mathcal{C}\left(\mathbf{x}_{s}, s, \mathbf{x}_{0}, 0\right)=\mathbb{E}\left[\delta U^{\alpha}\left(\mathbf{x}_{s}, s\right) \delta U^{\alpha}\left(\mathbf{x}_{0}, 0\right)\right]$ and $\delta \mathbf{U}$ is the fluctuating fluid velocity obtained from the solution of Eq. (6). The computation of $D_{h}$ is greatly simplified by iteratively inserting the solution of the tracer dynamics into Eq. (9), recalling that $\delta \mathbf{U}$ is small, and thus Taylor expanding around $\mathbf{x}_{0}$ (see $[58,59]$ ). At leading order, we obtain $D_{h} \simeq \frac{1}{3} \int_{0}^{\infty} d s \mathcal{C}\left(\mathbf{x}_{0}, s, \mathbf{x}_{0}, 0\right)$, where corrections due to higher-order correlations have been discarded in agreement with the approximations made in the kinetic approach. Given the solution of the linear dynamics, the diffusivity reads $D_{h} /\left(l v_{0}\right)=-\left(\rho \kappa_{n}^{2} / 45 \pi^{2}\right) \int_{0}^{\infty} d k A^{2}\left(\epsilon_{n} k\right) / \omega_{n}\left(k \epsilon_{n}\right)+$ $\left(\rho^{2} \kappa_{n}^{3} / 225 \pi^{2}\right) \int_{0}^{\infty} d k A^{2}\left(\epsilon_{n} k\right) / \omega_{n}^{2}\left(k \epsilon_{n}\right)$ and is approximated by [37]

$$
\frac{D_{h}}{D_{h}^{\text {free }}} \approx \frac{1}{2}\left[1+\frac{\rho_{c}}{\rho_{c} \mp \rho} \pm\left(\frac{\rho}{\rho_{c}}+\frac{\rho \rho_{c}}{\left(\rho_{c} \mp \rho\right)^{2}}\right)\right] .
$$

As in Eq. (8) the top (bottom) sign corresponds to pushers (pullers). In the low density limit, $D_{h} \rightarrow D_{h}^{\text {free }}+\mathcal{O}\left(\rho^{2}\right)$, where $D_{h}^{\text {free }} /\left(l v_{0}\right)=\left\langle U^{2}\right\rangle_{\mathrm{MF}} / 3 \lambda_{n} v_{0}^{2}=7 \rho \kappa_{n}^{2} /\left(2048 \epsilon_{n} \lambda_{n}\right)$ is the diffusivity obtained from discarding interactions between shakers. An interesting observation is that $D_{h}^{\text {free }}$ is, contrary to the variance of the fluid velocity, sensitive to the presence of self-propulsion: for shakers, $D_{h}^{\text {free }}$ diverges as $\epsilon_{n} \rightarrow 0$, while an analogous computation for swimmers gives $D_{h}^{\text {free }} / l v_{0}=\rho \kappa_{n}^{2} / 48 \pi$ in agreement with earlier theoretical predictions $[28,29,33-36]$. We have confirmed this difference of $D_{h}^{\text {free }}$ between shakers and swimmers through LB simulations at low density.

In Fig. 3, we show that the enhanced diffusivity of tracers measured in LB simulations is perfectly described by the kinetic theory even close to the onset of turbulence. We further observe that $D_{h}$ deviates from $D_{h}^{\text {free }}$ even for small densities of shakers, again highlighting the importance of correlations. Moreover, Eq. (10) correctly predicts how $D_{h}$ depends differently on $\rho$ for pushers and pullers—an effect

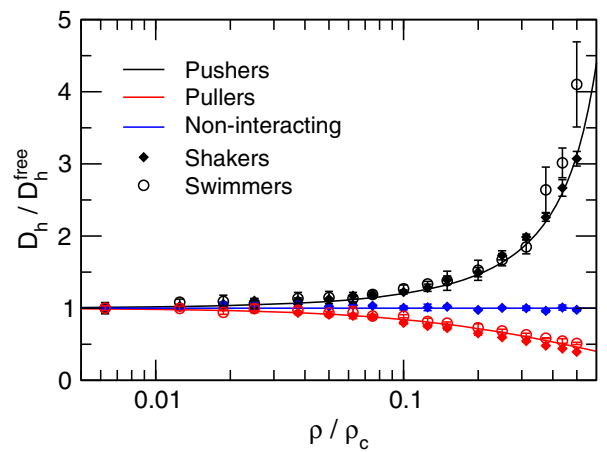

FIG. 3. Diffusivity $D_{h}$, normalized by its value $D_{h}^{\text {free }}$ in the noninteracting limit of passive tracers, as a function of the reduced swimmer density $\rho / \rho_{c}$. Symbols denote results from LB simulations of shakers or swimmers, and solid lines show predictions of the kinetic theory for the same parameters as in Fig. 2. Error bars represent one standard deviation, estimated by averaging over four separate LB runs. The theoretical results were obtained by numerically solving the integrals before Eq. (10). 
that has only briefly been discussed in the literature $[22,24,32,60,61]$.

An approach previously used by several authors [28,29,33-36] to predict $D_{h}$ considers tracer displacements due to scattering from a single swimmer. This leads to $D_{h} \simeq D_{h}^{\text {free }} \sim \rho \kappa_{n}^{2}$ and reflects the pusher-puller symmetry upon time-reversal in the Stokes equation. The extension of this argument to scattering by any finite number of swimmers presents a conceptual problem: the tracer displacement due to a scattering event by a collection of pushers can also be obtained in a suspension of pullers whose initial positions are set equal to the final positions of the pushers, with their orientations reversed. This argument thus suggests that $D_{h}$ should be equal for pushers and pullers at all densities, which is at odds with the numerical data in Fig. 3. The caveat in this argument is that it assumes a uniform sampling of initial conditions for the swimmers, while correlations between them will make some configurations more probable. When taken into account properly, these correlations break the pusher-puller symmetry even at moderate densities, as in Eq. (10).

Conclusions.-In this Letter, we have presented results from a novel kinetic theory and unprecedentedly large-scale particle-resolved simulations of microswimmer suspensions, describing quantitatively the fluctuations and correlations that arise at intermediate swimmer density. We have numerically shown that the collective motion in swimmer suspensions is the result of their rotational dynamics in the flow created by other swimmers while self-propulsion and spatial correlations play subdominant roles. We calculated the fluid velocity fluctuations and the enhanced diffusivity of tracer particles, and we found significant deviations from the mean-field predictions even at moderate swimmer densities. We demonstrated that swimmer-swimmer correlations are responsible for these deviations and should thus be taken into account well below the onset of bacterial turbulence. Understanding such correlations is a prerequisite for a deeper understanding of the turbulent state itself, in particular with regards to the presence or absence of a finite characteristic length scale in collective motion of microswimmers [5-8,13-15].

Discussions with Mike Cates and Joost de Graaf are kindly acknowledged. J. S. is financed by a grant from the Swedish Research Council (No. 2015-05449), C. N. by EPSRC Grant No. EP/J007404, R. W. N. by Intel through EPCC's Intel Parallel Computing Centre, and A. M. by EPSRC through Grant No. EP/I004262/1. C. N. acknowledges the hospitality provided by DAMTP, University of Cambridge, while part of this work was being done, and the support of Aide Investissements d'Avenir du LabEx PALM (ANR-10-LABX-0039-PALM). Research outputs generated through the EPSRC Grant No. EP/I004262/1 can be found at [62].

J. S. and C. N. contributed equally to this work. *joakim.stenhammar@fkem1.lu.se

cesare.nardini@gmail.com

†alexander.morozov@ph.ed.ac.uk

[1] S. Ramaswamy, Annu. Rev. Condens. Matter Phys. 1, 323 (2010).

[2] M. Marchetti, J. Joanny, S. Ramaswamy, T. Liverpool, J. Prost, M. Rao, and R. A. Simha, Rev. Mod. Phys. 85, 1143 (2013).

[3] M. E. Cates, Rep. Prog. Phys. 75, 042601 (2012).

[4] W. K. C. Poon, arXiv:1306.4799.

[5] C. Dombrowski, L. Cisneros, S. Chatkaew, R. E. Goldstein, and J. O. Kessler, Phys. Rev. Lett. 93, 098103 (2004).

[6] A. Sokolov, I.S. Aranson, J. O. Kessler, and R. E. Goldstein, Phys. Rev. Lett. 98, 158102 (2007).

[7] J. Gachelin, A. Rousselet, A. Lindner, and E. Clement, New J. Phys. 16, 025003 (2014).

[8] A. Creppy, O. Praud, X. Druart, P. L. Kohnke, and F. Plouraboué, Phys. Rev. E 92, 032722 (2015).

[9] T. Sanchez, D. T. N. Chen, S. J. DeCamp, M. Heymann, and Z. Dogic, Nature (London) 491, 431 (2012).

[10] D. L. Blair, T. Neicu, and A. Kudrolli, Phys. Rev. E 67, 031303 (2003).

[11] A. Bricard, J.-B. Caussin, N. Desreumaux, O. Dauchot, and D. Bartolo, Nature (London) 503, 95 (2013).

[12] J. Palacci, S. Sacanna, A. P. Steinberg, D. J. Pine, and P. M. Chaikin, Science 339, 936 (2013).

[13] A. Sokolov and I. S. Aranson, Phys. Rev. Lett. 109, 248109 (2012).

[14] J. Dunkel, S. Heidenreich, K. Drescher, H. H. Wensink, M. Bär, and R. E. Goldstein, Phys. Rev. Lett. 110, 228102 (2013).

[15] H. H. Wensink, J. Dunkel, S. Heidenreich, K. Drescher, R. E. Goldstein, H. Löwen, and J. M. Yeomans, Proc. Natl. Acad. Sci. U.S.A. 109, 14308 (2012).

[16] D. Saintillan and M. J. Shelley, Phys. Rev. Lett. 100, 178103 (2008).

[17] G. Subramanian and D. L. Koch, J. Fluid Mech. 632, 359 (2009).

[18] C. Hohenegger and M. J. Shelley, Phys. Rev. E 81, 046311 (2010).

[19] D. Saintillan and M. J. Shelley, C.R. Phys. 14, 497 (2013).

[20] D. Saintillan and M. J. Shelley, Phys. Rev. Lett. 99, 058102 (2007).

[21] C. W. Wolgemuth, Biophys. J. 95, 1564 (2008).

[22] D. Saintillan and M. J. Shelley, J. R. Soc. Interface 9, 571 (2012).

[23] E. Lushi and C. S. Peskin, Comput. Struct. 122, 239 (2013).

[24] D. Krishnamurthy and G. Subramanian, J. Fluid Mech. 781, 422 (2015).

[25] E. Lauga and T. R. Powers, Rep. Prog. Phys. 72, 096601 (2009).

[26] Y. Qian, P. R. Kramer, and P. T. Underhill, Phys. Rev. Fluids 2, 043104 (2017).

[27] X.-L. Wu and A. Libchaber, Phys. Rev. Lett. 84, 3017 (2000).

[28] G. Miño, T. E. Mallouk, T. Darnige, M. Hoyos, J. Dauchet, J. Dunstan, R. Soto, Y. Wang, A. Rousselet, and E. Clement, Phys. Rev. Lett. 106, 048102 (2011).

[29] A. Jepson, V. A. Martinez, J. Schwarz-Linek, A. Morozov, and W. C. K. Poon, Phys. Rev. E 88, 041002(R) (2013). 
[30] K. C. Leptos, J. S. Guasto, J. P. Gollub, A. I. Pesci, and R. E. Goldstein, Phys. Rev. Lett. 103, 198103 (2009).

[31] R. Jeanneret, D. O. Pushkin, V. Kantsler, and M. Polin, Nat. Commun. 7, 12518 (2016).

[32] H. Kurtuldu, J. S. Guasto, K. A. Johnson, and J. P. Gollub, Proc. Natl. Acad. Sci. U.S.A. 108, 10391 (2011).

[33] Z. Lin, J.-L. Thiffeault, and S. Childress, J. Fluid Mech. 669, 167 (2011).

[34] D. O. Pushkin and J. M. Yeomans, Phys. Rev. Lett. 111, 188101 (2013).

[35] T. Kasyap, D. L. Koch, and M. Wu, Phys. Fluids 26, 081901 (2014).

[36] A. Morozov and D. Marenduzzo, Soft Matter 10, 2748 (2014).

[37] See Supplemental Material at http://link.aps.org/ supplemental/10.1103/PhysRevLett.119.028005 for further details.

[38] The rotational dynamics of Eq. (1) corresponds to flowaligning swimmers and is valid in the limit $l \gg a$; the generalization to finite aspect ratios is straightforward and does not change the main conclusions drawn from the model.

[39] R. W. Nash, R. Adhikari, and M. E. Cates, Phys. Rev. E 77, 026709 (2008).

[40] R. W. Nash, R. Adhikari, J. Tailleur, and M. E. Cates, Phys. Rev. Lett. 104, 258101 (2010).

[41] Y. Hatwalne, S. Ramaswamy, M. Rao, and R. A. Simha, Phys. Rev. Lett. 92, 118101 (2004).

[42] R. Cortez, L. Fauci, and A. Medovikov, Phys. Fluids 17, 031504 (2005).

[43] R. Balescu, Statistical Dynamics: Matter Out of Equilibrium (Imperial College Press, London, 1997).

[44] C. W. Gardiner, Handbook of Stochastic Methods, 3rd ed. (Springer, Berlin, Heidelberg, 2004).
[45] D. R. Nicholson, Introduction to Plasma Theory (Wiley, New York, 1983).

[46] J. Heyvaerts, Mon. Not. R. Astron. Soc. 407, 355 (2010).

[47] A. Campa, T. Dauxois, and S. Ruffo, Phys. Rep. 480, 57 (2009).

[48] F. Bouchet, S. Gupta, and D. Mukamel, Physica (Amsterdam) 389A, 4389 (2010).

[49] C. Nardini, S. Gupta, S. Ruffo, T. Dauxois, and F. Bouchet, J. Stat. Mech. Theor. Exp. 2012, L01002 (2012).

[50] C. Nardini, S. Gupta, S. Ruffo, T. Dauxois, and F. Bouchet, J. Stat. Mech. Theor. Exp. 2012, P12010 (2012).

[51] This hypothesis is checked a posteriori showing that the final results keep the order of magnitude assumed.

[52] M. Doi and S. F. Edwards, The Theory of Polymer Dynamics (Oxford University Press, New York, 1988).

[53] We assume here $f=n / 4 \pi$, which simplifies significantly both the expressions for $C$ and for $L_{f}$ and which corresponds to a suspension that is isotropic and homogeneous on average. Their expressions for general $f$ are given in [37].

[54] C. Nardini, J. Stenhammar, and A. Morozov (to be published).

[55] We assume here $g(t=0)=0$. A different initial condition for $g$ only amounts to a transient, exponentially decaying contribution which does not affect the stationary properties.

[56] J.-L. Thiffeault, Phys. Rev. E 92, 023023 (2015).

[57] R. Kubo, Rep. Prog. Phys. 29, 255 (1966).

[58] F. Bouchet and T. Dauxois, J. Phys. Conf. Ser. 7, 34 (2005).

[59] F. Bouchet and T. Dauxois, Phys. Rev. E 72, 045103 (2005).

[60] P. T. Underhill, J. P. Hernandez-Ortiz, and M. D. Graham, Phys. Rev. Lett. 100, 248101 (2008).

[61] T. Ishikawa, J. T. Locsei, and T. J. Pedley, Phys. Rev. E 82, 021408 (2010).

[62] http://dx.doi.org/10.7488/ds/1703. 